\title{
Reflections on the DCT2 post at the University of Portsmouth Dental Academy
}

\author{
By Georgina Prosser, a Dental Officer working for the \\ Special Care Dental Service in North Devon, UK
}

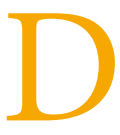
ental Core Training (DCT) posts are very popular with dental graduates as they provide exposure to different dental specialities, most notably oral and maxillofacial surgery, paediatric and restorative dentistry. There are a few split posts in existence that combine dental specialities along with experience in primary dental care. However, up until recently, there were no posts offering the opportunity of working in a primary care setting and also undertaking a postgraduate degree in research.

The DCT2 post at the University of Portsmouth Dental Academy, a collaboration between the University and Thames Valley and Wessex Health Education England, is the first of its kind and is comprised of part-time clinical activity in primary dental care and part-time research; more specifically time was allocated to the completion of a postgraduate Master's in Research (MRes) degree with the university. There were also opportunities to get involved with teaching undergraduate dental care professional students. Having just completed this unique post, I wanted to share my experiences in the hope of encouraging the creation of more posts of this nature.

In the first few years following graduation from dental school, my Dental Foundation
Training (DFT) and DCT1 jobs were heavily clinically orientated, and whilst my skills in operative dentistry improved, my proficiencies in critical appraisal and research certainly were not enhanced. Upon embarking on the research at the start of the post, I was therefore apprehensive but keen to immerse myself in the world of research and learning research methodology. Through completion of the structured one-year MRes degree and with the support of my supervisors, I was able to further my skills and knowledge in many aspects of conducting research. This included completion of a literature review and critical appraisal, writing a funding proposal, gaining ethical approval, conducting both quantitative and qualitative research, analysing the data and finally summarising it all in the form of a research manuscript. I was also involved in a vibrant journal club both presenting and supporting my fellow $\mathrm{PhD}$ and MRes student colleagues. I have been able to present my findings at two international conferences (unfortunately due to the COVID-19 pandemic - remotely) which was a great experience and allowed networking with individuals who shared an interest and expertise in the subject area of my research. I have just handed in my

\section{Liverpool student wins essay competition}

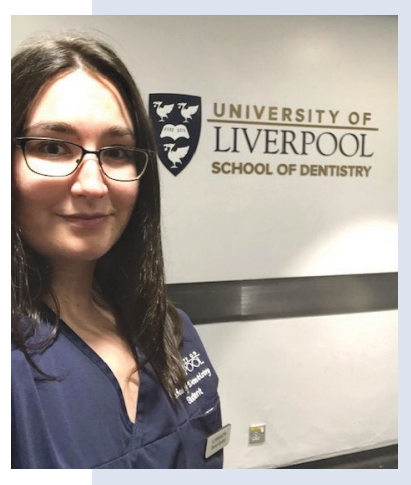

Aleksandra Krawczyk, a fifth-year dental student at the University of Liverpool, School of Dentistry, is the winner of the Keith Woods essay competition, awarded by the British Association for the Study of Community Dentistry (BASCD).

The annual essay competition is dedicated to the memory of Keith Woods, a long-standing member of the BASCD. The competition is open to any undergraduate studying dentistry, dental therapy, dental hygiene or dental nursing in the UK. The title for this year's essay was: 'What should the oral health care workforce look like in the UK by the middle of the twenty-first century to meet the needs of its population?'

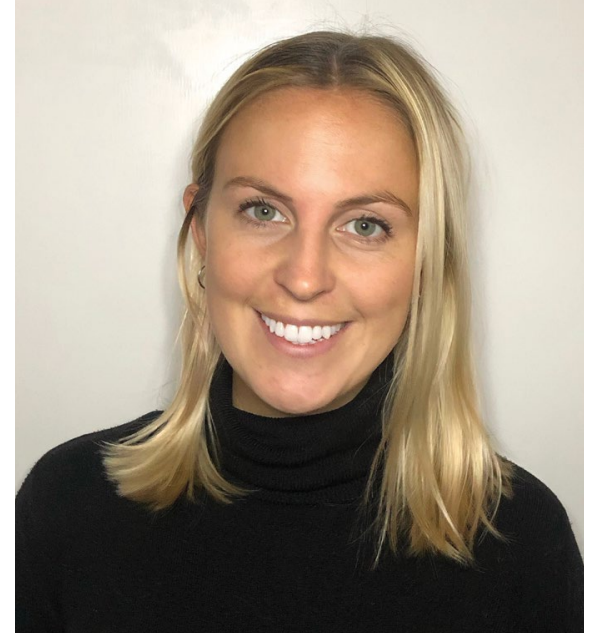

final assignment and submitted my research as two papers to two separate journals for publication.

Having come to the end of the degree and upon reflection of the year, I would highly recommend undertaking further training or even just seeking out opportunities to be involved in research. This is crucial to the future of the profession and, in my experience, has strengthened skills that can often be forgotten if only involved with the clinical side of dentistry. This DCT2 post offered the perfect mix of clinical training, which has been extremely beneficial in getting me back into the mindset of a general dental practitioner, alongside exploration of a different side of dentistry. I would implore the creation of similar posts in the future across the country.

\section{Death notice}

Julian Dominic James West died unexpectedly on 6 November, aged 57. A graduate of Newcastle Dental School, devoted son of Jim, brother to Barbara and Nigel, most loving uncle to William, Alistair, James, Harry and Philippa. A funeral was held on 26 November. Donations if desired to Horticap c/o J. E. Spence, Lawnswood Funeral Home, Otley Road, LS16 8AA, UK. 